\title{
Embolization of Type 1 Endoleak Due to Migration of Nellix Endograft System: Clinical Photos of Interest
}

\author{
Tal M Hörer MD PhD ${ }^{1,2}$ and David T McGreevy MD \\ 'Department of Cardiothoracic and Vascular Surgery, Faculty of Medicine and Health, Örebro University Hospital and \\ University, Sweden \\ ${ }^{2}$ Department of Surgery Faculty of Medicine and Health, Örebro University Hospital and University, Sweden
}

Keywords: Nellix; Embolization; Endoleak; Rupture

An 85-year-old male patient, 3 years after Nellix endograft implantation due to abdominal aortic aneurysm, presented to the clinic for annual follow-up with a huge endoleak secondary to aneurysm expansion and Nellix endograft migration. In order to prevent a rupture of the aortic aneurysm, and as the patient was not a candidate for open surgery, the endoleak was sealed with Ruby Coils (Penumbra, Alameda, USA) and Onyx (Medtronic, Santa Rosa, CA). Figure 1 shows the endoleak on computed tomography (CT; arrows mark the endoleaks). Figure $2 a-c$ shows the angiography procedure with Ruby Coils and Onyx filling the endoleak space. Figure $3 a, b$ shows the post-operative CT. The endoleak diminished on post-operative CT and was not detected by contrast enhanced ultrasound and the patient is planned for follow-up. Tight follow-up is recommended as this is a rescue procedure that we have used in several patients but long-time follow-up is unknown. This method has been used in symptomatic and ruptured aortic aneurysms with different endograft configurations.

\section{Corresponding author:}

David T McGreevy, MD, Department of Cardiothoracic and Vascular Surgery, Örebro University Hospital, SE-701 85 Örebro, Sweden.

Email: david.mcgreevy@regionorebrolan.se

(c) 2021 CC BY 4.0 - in cooperation with Depts. of Cardiothoracic/ Vascular Surgery, General Surgery and Anesthesia, Örebro University Hospital and Örebro University, Sweden

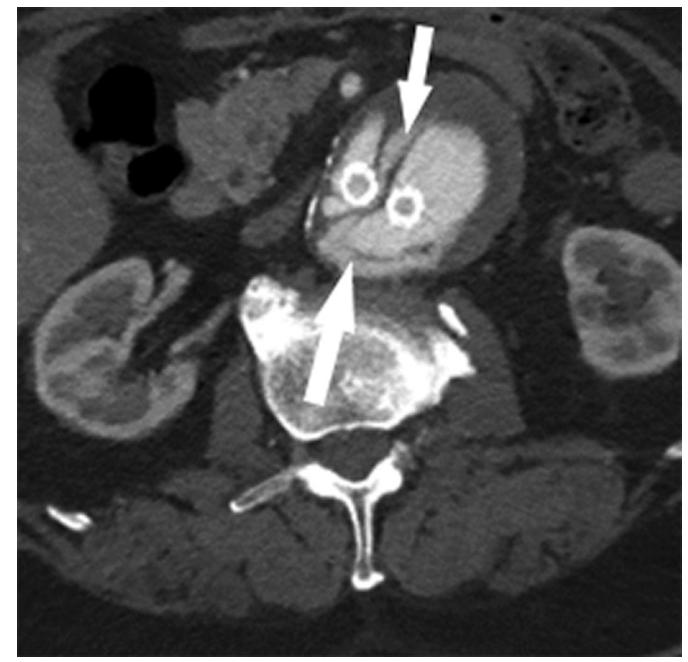

Figure 1

\section{Ethics Statement}

(1) All the authors mentioned in the manuscript have agreed to authorship, read and approved the manuscript, and given consent for submission and subsequent publication of the manuscript.

(2) The authors declare that they have read and abided by the JEVTM statement of ethical standards including rules of informed consent and ethical committee approval as stated in the article.

\section{Conflict of Interest}

The authors declare that they have no conflicts of interest. 

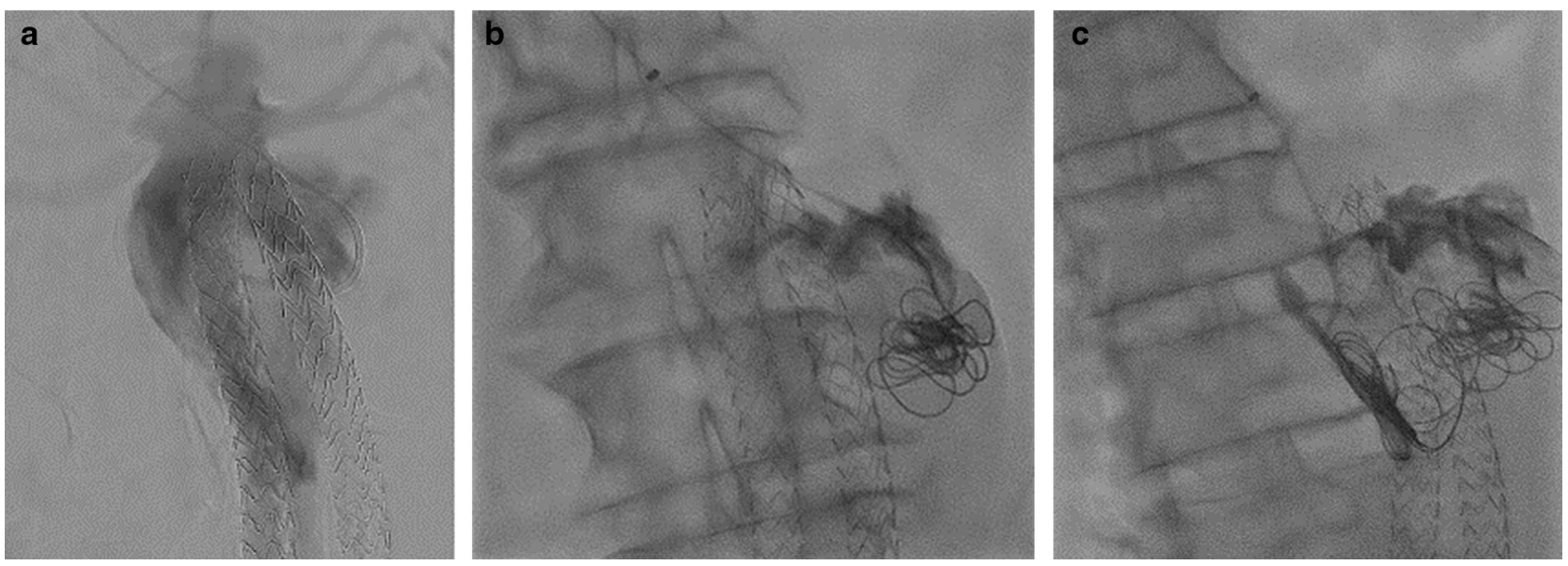

Figure 2
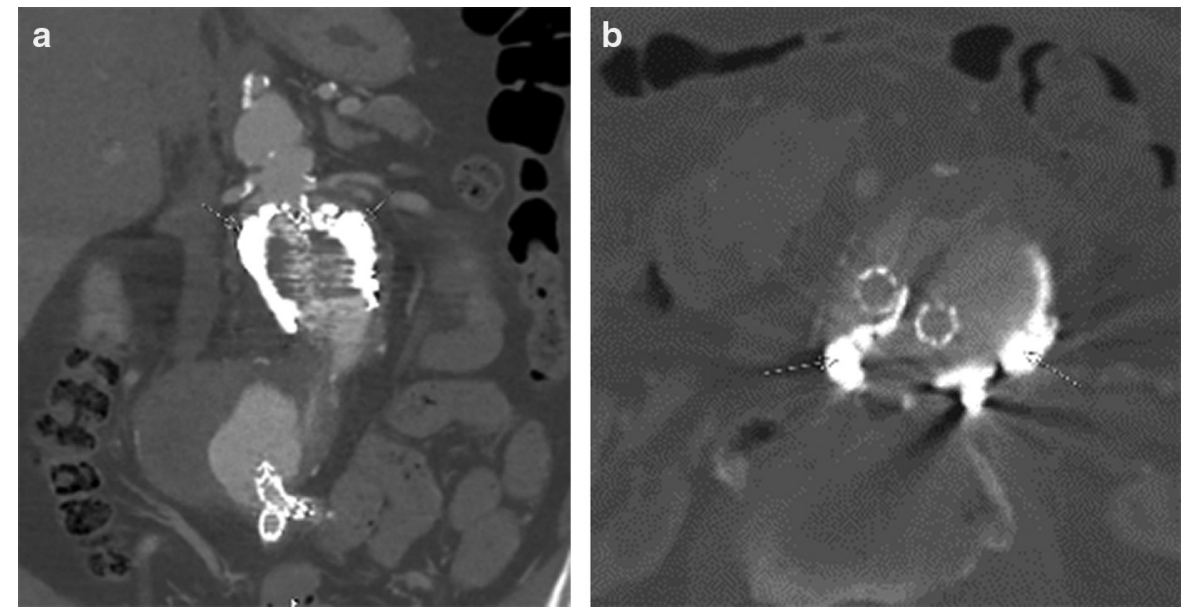

Figure 3

\section{Funding}

The authors received no financial support for the research, authorship, and/or publication of this article.

\section{Author Contributions}

TMH and DTM drafted, wrote and revised the manuscript. 